\title{
Review Paper: Introduction of Pediatric Balance Therapy in Children with Vestibular Dysfunction: Review of Indications, crossnark Mechanisms, and Key Exercises
}

Younes Lotfi ${ }^{1}$, Nima Rezazadeh ${ }^{2 *}$, Abdollah Moossavi ${ }^{1}$, Hojjat Allah Haghgoo ${ }^{3}$, Sedigheh Farokhi Moghadam ${ }^{3}$, Ebrahim Pishyareh $^{3}$, Enayatollah Bakhshi ${ }^{4}$, Reza Rostami ${ }^{5}$, Vahid Sadeghi ${ }^{6}$, Yousef Khodabandelou ${ }^{5}$

1. Department of Otolaryngology, School of Medicine, Iran University of Medical Sciences, Tehran, Iran.

2. Department of Audiology, University of Social Welfare and Rehabilitation Sciences, Tehran, Iran.

3. Department of Occupational Therapy, University of Social Welfare and Rehabilitation Sciences, Tehran, Iran.

4. Department of Statistics, University of Social Welfare and Rehabilitation Sciences, Tehran, Iran.

5. Department of Psychology, School of Behavioral Sciences and Mental Health, Tehran University of Medical Sciences, Tehran, Iran.

6. Department of Psychology, Faculty of Education and Psychology, Shahid Beheshti University, Tehran, Iran.

Citation: Lotfi Y, Rezazadeh N, Moossavi A, Haghgoo H, Farokhi Moghadam S, Pishyareh E, et al. Introduction of pediatric balance therapy in children with vestibular dysfunction: Review of indications, mechanisms, and key Exercises. Iranian Rehabilitation Journal. 2016; 14(1):5-14. http://dx.crossref.org/10.15412/J.IRJ.08140102

dol: $:$ http://dx.crossref.org/10.15412/J.IRJ.08140102

Article info:

Received: 14 Jul. 2015

Accepted: 15 Nov. 2015

\section{Keywords:}

Children, Vestibular

Rehabilitation, Balance,

Vestibular

\begin{abstract}
A B S T RACT
The vestibular system is important for the development of normal movement reactions, motion tolerance, and motor control for postural alignment, balance, and vision. A vestibular system that is damaged by disease or injury in childhood can have a major impact on a child's development. In addition, the emergence of vestibular lesions may also lead to cognitive deficits, including attention deficit. Despite the advances in testing and documentation of vestibular deficits in children, the vestibular problems continue to be an overlooked entity. Many children do not receive treatment that could significantly improve function and address the developmental delays caused by vestibular disorders.

Vestibular rehabilitation therapy (VRT) has been defined as an effective modality for most individuals with disorders of the vestibular or central balance system disorders. The basis for the success of VRT is the use of existing neural mechanisms in the human brain for adaptation, plasticity, and compensation. The vestibular system cannot be considered as a separate entity ignoring other balance subsystems. Hence, a modified VRT program, named pediatric balance therapy with special modifications in exercises, was developed for children with vestibular disorders, in accordance to the whole balance system.
\end{abstract}

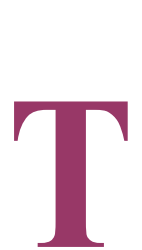

1. Introduction

he vestibular system develops relatively early during the gestation period and has been known as a dual-functioning system [1]. This system is composed of vestibulo-ocular and vestibulo-spinal subsystems, each represented by distinct tasks and testing methods. Labyrinthine reflexes, which are mediated by the vestibulo-spinal system, provide postural tone necessary for the emergence of early motor milestones (for e.g. rolling, sitting, standing) [2].

\section{* Corresponding Author:}

Nima Rezazadeh, PhD Candidate

Address: Department of Audiology, University of Social Welfare and Rehabilitation Sciences, Kodakyar Ave., Daneshjoo Blvd., Evin, Tehran, Iran.

Tel: +98 (21) 22180066

E-mail:nima_rz@yahoo.com 
Vestibular dysfunction in children may occur as a pure entity after audio-vestibular disorders. The incidence of peripheral vestibular disorders in children similar to that of the adults (e.g. Ménières disease, perilymphatic fistula, benign paroxysmal positional vertigo) has been reported [3-6]. Similarly, like adults with central nervous system impairment, the children with traumatic brain injury or other central nervous system insult may be expected to have central vestibular deficits [7]. However, investigations of vestibular dysfunction in children with such diagnoses are scarce [8-11]. Dysfunction may be found as comorbidity with developmental disorders [12]. Vestibular dysfunction has been reported in children with attention deficit, hyperactivity disorder [13], autism [14], cerebral palsy [15], Down 's syndrome [16], etc.

With respect to the lack of specialized vestibular programs in children with deep insight into the basic vestibular testing results and looking to whole balance system function, we tried to collect data to make a more comprehensive balance rehabilitation program in children with vestibular dysfunction.

\section{Adverse Effects of Vestibular Disorders in Children}

The vestibular system can get affected by various diseases during childhood, and this can have a major impact on a child's development [17]. Furthermore, cognitive vestibular interaction has been documented in many researches $[18$, 19]. The vestibular system plays a critical role in maintaining the balance function, and therefore, its inputs must be integrated with the highest brain functions [19]. Over the last decade, there has been a steady accumulation of evidence to suggest that vestibular lesions may also lead to cognitive deficits, including deficits in attention [18], and learning and memory [20] that are not necessarily directly attributable to the reflexive symptoms and perceptual abnormalities associated with the damage. Rather, there is increasing evidence that the loss of vestibular sensory information may alter, perhaps permanently, the way the cognitive processing areas of the brain integrate spatial and nonspatial information [18].

\section{VRT and PBT Program}

Vestibular Rehabilitation Therapy (VRT) has been defined as an effective modality for most individuals with disorders of the vestibular or central balance system [21]. The basis for the success of VRT is the use of existing neural mechanisms for adaptation, plasticity, and compensation. Specifically designed VRT exercise protocols take advantage of this plasticity of the brain to increase sensitivity and restore symmetry, which results in an improvement in vestibulo-ocular control, an increase in the gain of the vestibulo-ocular reflex (VOR), better postural strategies, and increased levels of motor control for movement [21].

As the vestibular system cannot be considered as a separate system ignoring other balance subsystems [22], we developed a modified VRT program for children with vestibular disorders taking into account the whole balance system. The modified system was named as pediatric balance therapy (PBT) with special modifications in exercises to use for the children of 4-12 age group.

The PBT program consists of different stages of balance training, gait exercises, and peripheral and central vestibular key exercises. So, children with vestibular dysfunction receive a comprehensive exercise battery to adapt the central nervous system to a sensory integrated movement planning. While this approach involves the active participation of the child and consists of complicated instructions, it is more practical to be used for children over 4 years of age. Focus of the exercises depends on the balance and vestibular assessment done by a trained audiologist or occupational therapist. Treatments can be delivered to the child via outpatient rehabilitation sessions or home exercises via trained parents. All balance and vestibular assessments can be used to measure the outcomes of the program. The average duration of the PBT program like any other VRT protocols [23] could range from 4 to 10 weeks. Further details will be discussed in PBT principles later.

\section{Assessment of Vestibular Functions in Children}

The functional integrity of the vestibular system is rarely tested in children. Hence, any impairment goes undetected and untreated. This situation may arise due to the children's inability to describe the symptoms and even its severity. One of the major difficulties in the identification of vestibular dysfunction in children is the unavailability or the omission of the use of traditional and more recently developed tests of vestibular function in this population [7]. Assessment of children must rely on functional and diagnostic test of $\mathrm{VO}^{1}$ and $\mathrm{Vsp}^{2}$ function and vestibular focused balance evaluation [7]. A previous study has reported the clinical measures of balance and functional assessments that have been normed and standardized for use in Iranian children with special disorders. These assessments include the functional reach test, the balance subtests of the Peabody developmental motor scale (PDMS), the locomotor subtest of the second edition PDMS (PDMS-II) [7], and the Bruininks-Oseretsky test of motor proficiency [24]. Care should

\footnotetext{
1. Vestibulo-ocular

2. Vestibulo-spinal
} 
be taken while selecting the tests because not all tests are appropriate for all age groups [7].

VO mechanisms can be examined by noting the presence of abnormal responses such as gaze-evoked nystagmus or gaze positional nystagmus, corrective saccades on the headthrust test [25], and visual stabilization during head rotation (DVA3 ${ }^{3}$ testing) [26]. In addition, the VO function can be assessed by rotational testing, which has been reported as a suitable test for evaluating pediatric population [27].

Vsp function can be reliably evaluated by DPT component of posturography [28] and VEMP testing, which has been shown as an applicable test in children [29]. DPT [7] also can be used beside oculomotor testing of Videonystagmography [30] to determine whether neurological dysfunction is evident along with its level in the pediatric population.

\section{PBT is Indicated for the Following Conditions}

Like any VRT program [23], children with the following conditions will be indicated for PBT program:

\section{Stable vestibular lesion}

PBT is indicated by any condition characterized by a stable vestibular deficit, in which evaluation reveals no evidence of a progressive process and the patient's natural compensation process appears to be incomplete.

Central lesions or mixed central and peripheral lesions

Patients with stable CNS lesions or mixed central and peripheral lesions should not be excluded from treatment, although their prognoses are likely to be more limited than the average patient with a stable peripheral injury.

\section{Neurological disorders or conditions}

Conditions like cerebral palsy, $\mathrm{ADHD}^{4}$, autism, hydrocephalus, a posterior brain tumor, or Wallenberg syndrome (caused by a stroke from a blockage in the vertebral or posterior inferior cerebellar artery of the brain stem).

\section{Situations with Non-indication of PBT}

Habituation will be almost impossible for an unstable lesion [31], and PBT is generally not applicable for children with unstable dysfunctions, such as Ménières disease. Other medical or surgical approaches should be considered in these cases.

\section{Dynamic Visual Acuity}

4. Attention Deficit And Hyperactivity Disorder

\section{Principles of PBT}

The goals of PBT are 1) Enhancing gaze stability, 2) Enhancing postural stability, and 3) Improving daily living activities. Special issues should be considered in PBT program such as:

- While the exercises need the active participation of the child, PBT could be used for any child over 4 years of age.

- Children should perform exercises for gaze stability four to five times daily for a total of 20-40 minutes/day, in addition to 20 minutes of balance and gait exercises [7].

- The choice of exercises is guided by both the child's symptoms and related vestibular assessment results [32]. Exercises must start with postural and gait exercises (Exercises 1-2 in the following section) within the first $20 \mathrm{~min}$ for all children, and specialized exercises will be selected based on child's vestibular function (Exercises 3-5 in the following section).

- Children must be evaluated by the therapist regarding their responses to vestibular stimulations. Children with exaggerated reactions (Hyper sense) should receive mild stimulations while children with a high tendency for vestibular stimulations (Hypo sense) should receive more intense stimulations [33].

- To maximize the child's participation and cooperation, the use of toys, games, and other items to facilitate visual tracking, or the use of swings to provide the movement during visual stabilization activities are considered important [7].

- Exercise duration is short (1-2 min), and the exercise intensity is defined according to the symptoms [32].

- Habituation and substitution exercises are repetitive (8-10 repetitions for exercises to stabilize gaze) [32].

- Vestibular rehabilitation therapy should be implemented with variable frequencies, with the performance of numerous different suitable tasks for children [32].

- While performing the exercises, stress symptoms need to be searched out, such as crying, blushing or fearing. The practice session needs to be ended with the appearance of any of the mentioned symptoms [33].

- Children with a central or mixed lesion expect a prolonged period of therapy, but the outcome does not vary with the location [34]. Those with mixed lesion sites may require longer therapy. In addition, children 


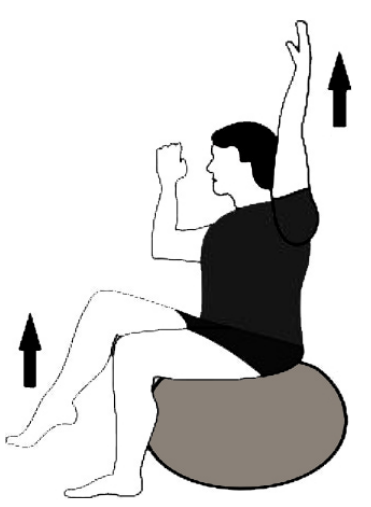

Figure 1. $\mathrm{CP}$ ball exercise.

Iranian Rehabilitation Journa

with a pure central lesion demonstrated a trend for a more successful therapy outcome compared to those with mixed lesions [35].

\section{Key Exercises}

Overall balance and gate exercises for children with vestibular dysfunction

\section{Trampoline}

- Ask the child to jump on a trampoline while he/she rotates around himself/herself [33].

- Ask the child to jump up and down on the trampoline [33]

Note: If the child loses his/her balance, make him/her stand in the center of the trampoline and ask them to put their hands on their waist [33].

\section{CP ball}

- Ask the child to sit in the center of the ball, opposite arm and leg lifted, hold for 15 seconds, then switch side with both eyes open and closed [33, 36] (Figure 1).

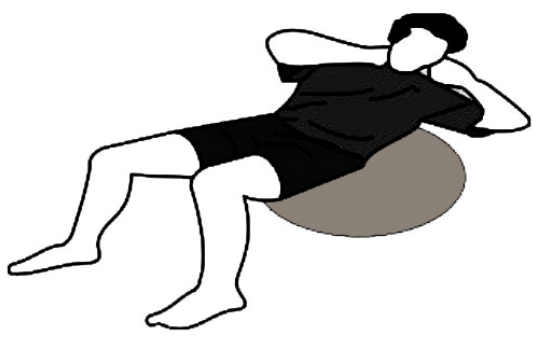

Figure 3. $\mathrm{CP}$ ball exercise.

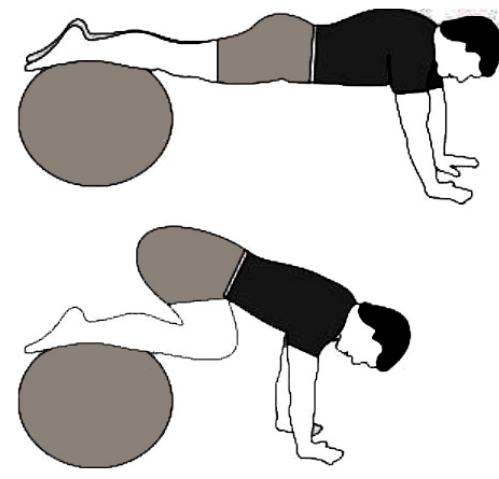

Figure 2. CP ball exercise

Iranian Rehabilitation Journal

- Place the child prone on CP ball and roll-up, so that his/her hands reach the ground and ask him/her to get up and return to the first position. Repeat it for $15 \mathrm{sec}-$ onds [33, 37] (Figure 2).

- Place the child supine on CP ball, diagonal curl-up, hips on ball, switch sides, each repeated for 15 seconds [36] (Figure 3).

- Place the child prone on CP ball, opposite arm and leg raised, ask him to hold for 15 seconds, and then switch sides [33, 37] (Figure 4).

- Place the child on CP ball, ask him/her to walk on the ball back and forth, and ask to repeat it for 15 seconds [37] (Figure 5).

- Place the child supine on CP ball, opposite arm and leg raised, hold for 15 seconds, and then switch sides [36] (Figure 6).

- Put the child supine on the ground, ask him/her to lift his/her pelvis for 15 seconds [36] (Figure 7).

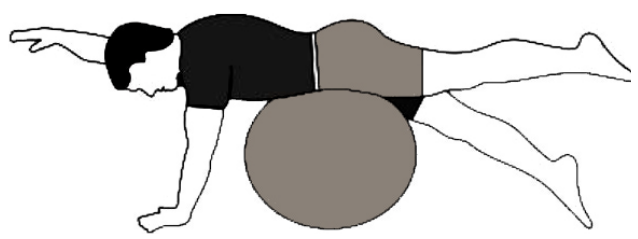

Iranian Rehabilitation Journal

Figure 4. $\mathrm{CP}$ ball exercise. 


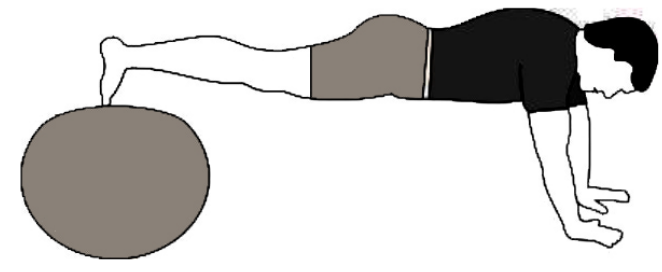

Ilranian Rehabilitation Journal

Figure 5. CP ball exercise.

\section{Balance board}

- Ask the child to stand on a balance board and hold on for 10 seconds [33].

- Ask the child to be on the balance board in quadruped position and hold on for 10 seconds [33].

- Ask the child to be on the balance board in kneeling position and hold on for 10 seconds [33].

\section{Rotating board}

- Ask the child to stand on the rotating board and hold on for 10 seconds [33].

- Ask the child to be on the rotating board in quadruped position and hold the position for 10 seconds [33].

- Ask the child to be on the rotating board in kneeling position and hold the position for 10 seconds [33].

\section{Scooter}

- Ask the child to sit on a scooter while the hands are cross around the chest and move in a straight line (5

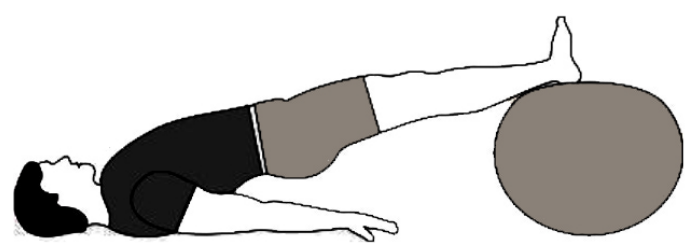

Figure 7. $\mathrm{CP}$ ball exercise.

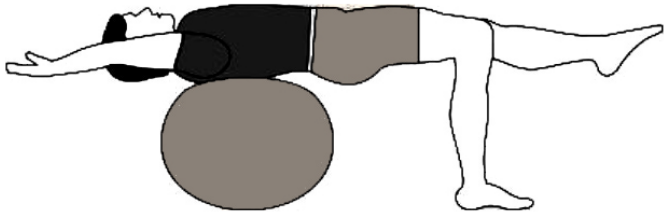

Iranian Rehabilitation Journa

Figure 6. $\mathrm{CP}$ ball exercise.

meters), then stop, and again move back on the same route (A total of 10 roles) [33].

- Place the child prone on a scooter, and ask him/her to move in the direction of rotation. After 5 to 10 times, change the rotation direction [33].

\section{Fencing balance}

- Ask the child to walk on fencing balance to the front, rear and sides [33].

Swing

- Ask the child to sit on a swing and move him/her. Initially, move linearly (Front and rear, left and right), and then begin rotational movement [33].

\section{Rocking T}

- The child is tied to a T-bar firmly. The bar is rotated approximately 5-10 times [33].

\section{Obstacle}

- Ask the child to jump over obstacles of various heights [33].

\section{Rolling}

- Ask the child to move across the floor, forward or backward, with the arms extended straight overhead and the legs held straight [33].

\section{Blanket}

- The child lies along one edge of the blanket and grasps the edge. Then rolls up in the blanket toward the edge approximately 5-10 times [33]. 

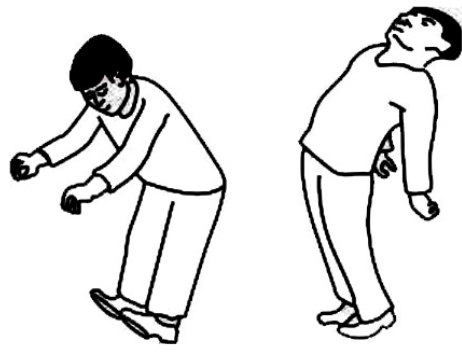

Figure 8. Postural stability exercises.

Iranian Rehabilitation Journa

\section{Walk the spiral trails}

- Ask the child to travel through different spiral paths [33]

\section{Running through routes spiral}

- The child is asked to run in spiral paths [33].

\section{Moving backward}

- The child is asked to move backward on a straight line [33].

\section{Difficult moving backward}

- The child is asked to move backward on a spiral path [33].

Postural Stability exercises for children with vestibular dysfunction

- Ask the child to stand on one leg. Ask him/her to do it for 15 seconds. Switch to the other leg (one of the Cawthorne-Cooksey exercise) [34]

- Ask the child to stand with the feet heel-to-toe with both arms extended for 15 seconds. Ask him/her to switch to the other leg [34].

- Sway back and forth. Locate the child behind a chair and before a wall. This prevents the child from falling. Ask the child to bend and move the center of body backward with his/her toes up. Now ask him/her to bend backward and move the center of his/her body forward with the heels up. Repeat it for 10 times [34] (Figure 8).

- Ask the child to march in place. Ask him/her to do it for 15 seconds with eyes open and closed [34].

- Ask the child to walk straight while he/she is turning his/her head. It is better to do it near a wall for needed help. Ask him/her to do it for 15 seconds [7].

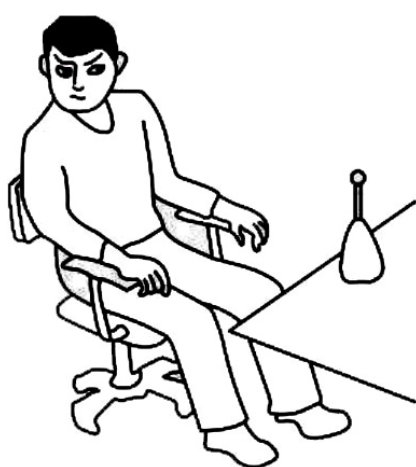

Iranian Rehabilitation Journal

Figure 9. Peripheral vestibular exercises.

- Ask the child to walk on a hard surface for 10 seconds. Then ask him/her to walk back for 10 seconds. Now ask him/her to try it on foam.

- Ask the child to walk around a big circle for 15 seconds. Time by time, make the circle smaller and smaller [7].

- Ask the child to stand with his/her feet shoulder-width apart with eyes open looking at a target on a wall for 20 seconds. Now ask him to narrow his/her base of support to semi-heel-to-toe position while he/she is looking at the target. Now ask him/her to do it while his/her eyes are closed [7].

Peripheral vestibular exercises for children with Unilateral vestibular dysfunction (focused on adaptive strategies)

- All exercises mentioned in part 1 and 2 needs to be followed.

- Ask the child to move his/her head horizontally with different speeds while he/she is looking at a target in front for 1 minute. Now ask him/her to move his/her head vertically [34] (Figure 9).

- Now ask the child to look at his/her thumb while he/ she is moving his head and trunk together on a rotational chair. Do it for 30 seconds [34] (Figure 10).

- Ask the child to look at a target on a wall while walking, moving his/her head horizontally for 15 seconds Now ask him/her to do it while he/she is moving his/ her head vertically [34].

- Ask the child to sit down on a cp ball and look at a target in front while he/she is moving his body up and down for 1 minute (Otolith Stimulation) [7]. 


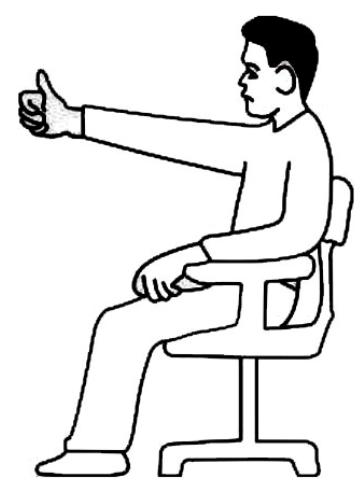

IIranian Rehabilitation Journal

Figure 10. Peripheral vestibular exercises.

Peripheral vestibular exercises for children with Bilateral Vestibular Dysfunction (focused on adaptive and substitutive strategies)

- All exercises in part 1-2 and part 3 (to facilitate gaze stabilization).

- Put 2 targets horizontally in front of a child on the wall. Ask him to look at one target for 10 seconds. Afterward, ask him to look at the next target with head fixed. Now ask him/ her to move his head toward the second target. Do it for 2-3 minutes. Now put the targets vertically and repeat the exercise. Ask the child to do it faster time by time [23]. This active eye-head movement will facilitate the saccadic or smooth pursuit strategies and central pre-programing [7].

- Ask the child to look at different targets with high eye speed with eyes fixed for 30 seconds (this exercise will facilitate gaze stabilization using saccadic eye movements) [38].

- Ask the child to stand with open eyes while he/she is opening his/her arms in front for 20 seconds. Now ask $\mathrm{him} / \mathrm{her}$ to do it with eyes closed (simultaneous use of vestibular and somatosensory inputs) [38].

- Now ask him/her to repeat the above exercise on a foam with eyes opened and closed for 20 seconds (Simultaneous use of vestibular and visual inputs) [38].
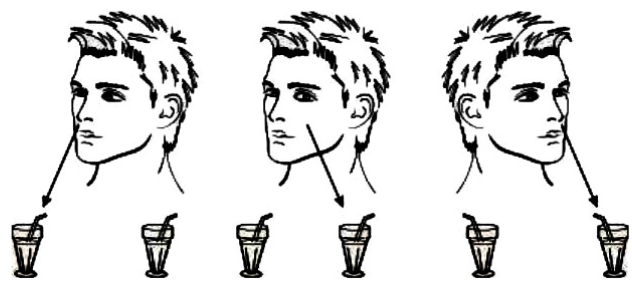

Figure 11. Saccade and VOR.
- Ask the child to sit in front of a target and look at it. Now ask him to close his/her eyes remembering the target in his/her mind. He has to be on the target when he/she opens his/her eyes (to facilitate central programming). Do it five times [7].

- Ask the child to walk around the room while he/she suddenly move his/her head and trunk to the left and right side. Ask him/her to do it for 10 times [23].

Eye movement exercises for children with central or mixed peripheral and central Vestibular lesions

- All exercises of parts 1 and 2 are performed.

Gaze

Ask the child to keep his/her eyes still on a target for 20 seconds.

Saccade

Ask the child to keep the head still and move only the eyes. Place two targets close enough together while the child is looking directly at one. Ask him/her to look at one target and quickly look at the other target, without moving the head. These movements are repeated several times (one of the Cawthorne-Cooksey exercises) [34].

\section{Saccade and VOR}

Ask the child to look directly at a target, ensuring that his head is aligned with the target; then ask him/her to look at the other target; and afterward ask him/her to turn his/her head to the other target [35] (Figure 11).

\section{Standard pursuit}

Ask the child to look at your hand in front of him/her while you are moving it slowly for 40 seconds. Move it with different speed, horizontally and vertically.

\section{Imagery pursuit (remembered target exercise)}

Ask the child to look directly at a target. Then ask him/her to close the eyes and move the head slowly turning away from
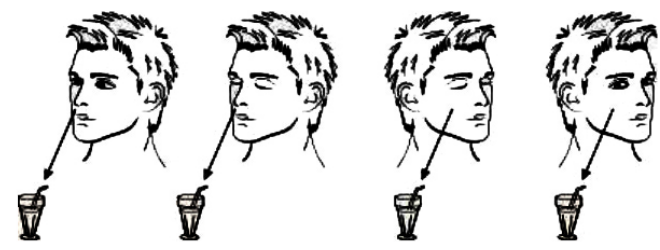

Iranian Rehabilitation Journal

Figure 12. Imagery pursuit. 
the target while imagining that one is still looking at the target Then tell him/her to open the eyes and check whether the target is kept in focus. If not, adjust the gaze on the target. Do it for 5 times. Repeat in the opposite direction [35] (Figure 12).

\section{Optokinetic exercises}

Use different optokinetic video format on the computer screen. Ask the child to follow the white and black bars to the end and back to the center. Do it for two minutes. Use bars with different speeds. First, use horizontal bars followed by vertical ones.

\section{Conclusion}

A well-designed and strategic PBT program in children might bring a significant improvement in child's learning abilities, balance, development, and self-confidence. However, the effectiveness of such a program depends on the interest level of the children affected with vestibular dysfunction.

\section{Acknowledgements}

The current study was part of $\mathrm{PhD}$ dissertation project in Audiology that was approved by the local ethical committee of the University of Social Welfare and Rehabilitation Sciences (USWR) with ethical code of USWR.REC.1392.114. The research has received no specific grant from any funding agency, commercial or not-for-profit sectors.

\section{Conflict of Interests}

The authors declared no conflict of interests.

\section{References}

[1] Hayes SH, Ding D, Salvi RJ, Allman BL. Anatomy and physiology of the external, middle and inner ear. In: Gastone GC, editor. Handbook of Clinical Neurophysiology: New York: Elsevier; 2013, p. 3-23.

[2] Wiener-Vacher SR, Toupet F, Narcy P. Canal and otolith vestibulo-ocular reflexes to vertical and off vertical axis rotations in children learning to walk. Acta Oto-Laryngologica. 1996; 116(5):657-65.

[3] Buchman CA, Joy J, Hodges A, Telischi FF, Balkany TJ. Vestibular effects of cochlear implantation. Laryngoscope. 2004; 114(103):1-22

[4] D’Agostino R, Melagrana A, Taborelli G. Benign positional paroxysmal vertigo of horizontal semicircular canal in the child: case report. International Journal of Pediatric Otorhinolaryngology. 2003; 67(5):549-51.
[5] Monobe H, Murofushi T. Vestibular neuritis in a child with otitis media with effusion: clinical application of vestibular evoked myogenic potential by bone-conducted sound. International Journal of Pediatric Otorhinolaryngology. 2004; 68(11):1455-458.

[6] See GB, Mahmud MR, Zurin AA, Putra SH, Saim LB. Vestibular nerve section in a child with intractable Meniere's disease. International Journal of Pediatric Otorhinolaryngology. 2002; 64(1):61-64.

[7] Herdman SJ. Vestibular rehabilitation. $3^{\text {rd }}$ ed. Philadelphia: F.A. Davis; 2007

[8] Brogren E, Hadders-Algra M, Forssberg H. Postural control in children with spastic diplegia: muscle activity during perturbations in sitting. Developmental Medicine and Child Neurology. 1996; 38(5):379-88.

[9] Bundy AC, Fisher AG, Freeman M, Lieberg GK, Izraelevitz TE. Concurrent validity of equilibrium tests in boys with learning disabilities with and without vestibular dysfunction. American Journal of Occupational Therapy. 1987; 41(1):28-34.

[10] Horak FB, Shumway-Cook A, Crowe TK, Black FO. Vestibular function and motor proficiency of children with impaired hearing, or with learning disability and motor impairments. Developmental Medicine and Child Neurology. 1988; 30(1):64-79

[11] Reid DT, Sochaniwskyj A, Milner M. An investigation of postural sway in sitting of normal children and children with neurological disorders. Physical \& Occupational Therapy in Pediatrics. 1991; 11(1):19-36.

[12] Jahn K. Vertigo and balance in children-diagnostic approach and insights from imaging. European Journal of Paediatric Neurology. 2011; 15(4):289-94.

[13] Shum SB, Pang MY. Children with attention deficit hyperactivity disorder have impaired balance function: involvement of somatosensory, visual, and vestibular systems. Journal of Pediatrics. 2009; 155(2):245-49.

[14] Greffou S, Bertone A, Hahler EM, Hanssens JM, Mottron L, Faubert J. Postural hypo-reactivity in autism is contingent on development and visual environment: a fully immersive virtual reality study. Journal of Autism and Developmental Disorders. 2012; 42(6):961-70.

[15] Gatica VF, Irene Velasquez S, Mendez GA, Guzman EE Manterola CG. Differences in standing balance in patients with cerebral palsy and typically developing children. Biomedica: Revista del Instituto Nacional de Salud. 2014; 34(1):102-09.

[16] Inagaki T, Morita N, Cureoglu S, Schachern PA, Nomiya S, Nomiya $R$, et al. Peripheral vestibular system in Down syndrome: quantitative assessment of vestibular histopathology. Otolaryngology Head and Neck Surgery. 2011; 144(2):280-83.

[17] Suarez H, Angeli S, Suarez A, Rosales B, Carrera X, Alonso R. Balance sensory organization in children with profound hearing loss and cochlear implants. International Journal of Pediatric Otorhinolaryngology. 2007; 71(4):629-37.

[18] Smith PF, Zheng Y, Horii A, Darlington CL. Does vestibular damage cause cognitive dysfunction in humans? Journal of Vestibular Research. 2005; 15(1):1-9. 
[19] Guidetti G. The role of cognitive processes in vestibular disorders. Hearing, Balance and Communication. 2013; 11(1):3-35.

[20] Smith PF, Haslett S, Zheng Y. A multivariate statistical and data mining analysis of spatial memory-related behaviour following bilateral vestibular loss in the rat. Behavioural Brain Research. 2013; 246:15-23.

[21] Morozetti PG, Gananca CF, Chiari BM. Comparison of different protocols for vestibular rehabilitation in patients with peripheral vestibular disorders. Jornal da Sociedade Brasileira de Fonoaudiologia. 2011; 23(1):44-50.

[22] Cesarani A, Alpini D, Capobianco S. The MCS (Mechanic, Cybernetic and Synergetics) method in vestibular rehabilitation. In: Cesarani A, Alpini D, editors. Vertigo and Dizziness Rehabilitation. Berlin Heidelberg: Springer-Verlag Berlin Heidelberg; 2004, p. 1-22.

[23] Tee LH, Chee NW. Vestibular rehabilitation therapy for the dizzy patient. Annals of the Academy of Medicine, Singapore. 2005 ; 34(4):289-94.

[24] Jafari Z, Malayeri S, Rezazadeh N, Haji Heydari F. Static and dynamic balance in congenital severe to profound hearing-impaired children. Audiology. 2011; 20(2):102-12.

[25] Schubert MC, Tusa RJ, Grine LE, Herdman SJ. Optimizing the sensitivity of the head thrust test for identifying vestibular hypofunction. Physical Therapy. 2004; 84(2):151-58.

[26] Rine RM, Braswell J. A clinical test of dynamic visual acuity for children. International Journal of Pediatric Otorhinolaryngology. 2003; 67(11):1195-201.

[27] Salami A, Dellepiane M, Crippa B, Barettini L, Mora R. Visual-vestibular interaction test in the diagnosis of vertigo in children. International Journal of Pediatric Otorhinolaryngology. 2008; 72(1):1-7.

[28] Medeiros IR, Bittar RS, Pedalini ME, Lorenzi MC, Kii MA Formigoni LG. Evaluation of the treatment of vestibular disorders in children with computerized dynamic posturography: preliminary results. Jornal de Pediatria. 2003; 79(4):33742.

[29] Rezazadeh N, Ahadi M, Talebi H, Gharib M, Yazdani N, Shahrokhi A. [Vestibular evoked myogenic potentials (VEMPs) in children (Persian)]. Journal of Rehabilitation. 2011; 11(5):21-25.

[30] Rosenhall U, Johansson E, Gillberg C. Oculomotor findings in autistic children. Journal of Laryngology and Otology. 1988; 102(5):435-39.

[31] Deveze A, Bernard-Demanze L, Xavier F, Lavieille JP, Elziere M. Vestibular compensation and vestibular rehabilitation: current concepts and new trends. Clinical Neurophysiology. 2014; 44(1):49-57.

[32] Boyera FC, Percebois-Macadréa L, Regrainb E, Lévêquec M, Taïar R, Seidermannc L, et al. Vestibular rehabilitation therapy. Clinical Neurophysiology. 2008; 38(6):479-87. doi: 10.1016/j.neucli.2008.09.011

[33] Cheatum BA, Hammond AA. Physical activities for improving children's learning and behavior: a guide to sensory motor development. Champaign: Human Kinetics; 2000.
[34] Han BI, Song HS, Kim JS. Vestibular rehabilitation therapy: review of indications, mechanisms, and key exercises. Journal of Clinical Neurology. 2011; 7(4):184-96.

[35] Shepard NT, Telian SA. Programmatic vestibular rehabilitation. Otolaryngology Head and Neck Surgery. 1995; 112(1):173-82.

[36] Willardson JM. Core stability training: applications to sports conditioning programs. Journal of Strength and Conditioning Research. 2007; 21(3):979-85.

[37] Queiroz BC, Cagliari MF, Amorim CF, Sacco IC. Muscle activation during four Pilates core stability exercises in quadruped position. Archives of Physical Medicine and Rehabilitation. 2010; 91(1):86-92.

[38] Gill-Body KM, Krebs DE, Parker SW, Riley PO. Physical therapy management of peripheral vestibular dysfunction: two clinical case reports. Physical Therapy. 1994; 74(2):129-42. 
